\title{
Théologiques
}

\section{La souffrance rédemptrice : légitimation ou subversion religieuse de la violence?}

\section{Jean-Guy Nadeau}

Volume 13, numéro 2, automne 2005

Violence et souffrance rédemptrices

URI : https://id.erudit.org/iderudit/013602ar

DOI : https://doi.org/10.7202/013602ar

Aller au sommaire du numéro

Éditeur(s)

Faculté de théologie et de sciences des religions, Université de Montréal

ISSN

1188-7109 (imprimé)

1492-1413 (numérique)

Découvrir la revue

Citer ce document

Nadeau, J.-G. (2005). La souffrance rédemptrice : légitimation ou subversion religieuse de la violence ? Théologiques, 13(2), 5-19.

https://doi.org/10.7202/013602ar

Tous droits réservés $\subset$ Faculté de théologie et de sciences des religions, Université de Montréal, 2006
Ce document est protégé par la loi sur le droit d'auteur. L'utilisation des services d'Érudit (y compris la reproduction) est assujettie à sa politique d'utilisation que vous pouvez consulter en ligne.

https://apropos.erudit.org/fr/usagers/politique-dutilisation/ 


\title{
La souffrance rédemptrice: légitimation ou subversion religieuse de la violence?
}

\author{
Jean-Guy NADEAU \\ Faculté de théologie et de sciences des religions \\ Université de Montréal
}

La modernité avait cru mettre fin à la légitimation religieuse de la violence. Mais voici que le nom de Dieu est toujours pris à témoin par les puissants comme par les impuissants de ce monde, légitimant par exemple le recours à la violence dans la résolution des conflits internationaux. La plupart du temps, les leaders des grandes religions rejettent ces légitimations, arguant par exemple de la place centrale de l'amour, de la paix ou de la compassion dans leurs textes fondateurs. Et pourtant, la violence n'est pas exclue de ces récits fondateurs où elle occupe même une place majeure, comme le montrent les actes de divinités - y compris dans la Bible et la tradition chrétienne - qui n'hésitent pas à user de la violence pour parvenir à leurs fins. Or, la violence implique la souffrance de ceux et celles qui en sont l'objet, et cela trop souvent comme victimes innocentes, victimes dont la figure de Jésus crucifié constitue en christianisme l'icône par excellence. Le christianisme, en effet, affirme la valeur salutaire et rédemptrice de cette souffrance, ce qui a pour conséquence de «sauver» la souffrance elle-même en lui donnant sens, particulièrement dans l'affirmation de la participation de nos souffrances à celles du Crucifié, mais a aussi pour effet de légitimer la souffrance, voire la violence qui en est trop souvent la source. La question est délicate dans la mesure où le salut constitue le cœur du message chrétien alors que la souffrance du Christ en est le moyen. Or, la valeur rédemptrice de la souffrance fait aujourd'hui problème à plusieurs, alors qu'elle en aide toujours d'autres à mieux vivre leur souffrance.

En fait, le problème n'est peut-être pas tant de considérer la souffrance comme salvifique que de la considérer comme rédemptrice, c'est-à-dire liée à l'expiation, et de la situer dans le plan divin. Que la souffrance sauve, nous en faisons chaque jour l'expérience, d'abord à partir des avertisse- 
ments que la douleur nous envoie. Nous en faisons aussi l'expérience quand nous voudrions nous substituer - j'utilise sciemment le terme - à l'enfant qui a mal et prendre sur nous sa douleur. Ou encore quand les nouvelles télévisées nous apprennent qu'un tel s'est constitué ou a offert de se constituer otage à la place d'un autre. Le savent aussi les victimes de torture qui gardent le silence le plus longtemps possible pour permettre à leurs compagnons et compagnes de se mettre en sécurité. Plusieurs, enfin, acceptent de souffrir pour des causes ou des gens dans lesquels ils croient, et on peut penser aux soldats ou militants sur les divers champs de bataille de l'histoire. Il arrive alors que la souffrance des uns s'avère historiquement salutaire pour les autres. Mais c'est autre chose d'inscrire cette souffrance dans un cadre métaphysique et de l'attribuer à la volonté de Dieu.

Dans ces pages introductives, nous voudrions présenter quelques-unes des questions soulevées par notre sujet, rappeler quelques éléments de la position classique souvent prise à partie par nos contemporains et réfléchir à la question du sens des souffrances humaines au regard de cette tradition et de quelques-unes de ses relectures contemporaines. Enfin, nous présenterons les contributions qui constituent ce numéro de Théologiques.

\section{Du scandale de l'événement au scandale du sens}

La souffrance et la mort ignominieuses de Jésus en croix constituaient un scandale pour la foi et la culture des premiers chrétiens. D'où la nécessité de les intégrer dans un narratif religieux signifiant où elles ont trouvé de la valeur et perdu en quelque sorte leur caractère scandaleux. Donner sens à l'événement, en effet, c'est souvent l'inscrire dans un narratif établi ou dans les cadres culturels ambiants et, ce faisant, en juguler le scandale (Ricœur 1991). C'est ainsi que le christianisme a thématisé comme rédemptrice la souffrance du Sauveur crucifié, la considérant comme un sacrifice dont il était aussi bien le Grand-Prêtre que la victime, bien que sa mort fut le fruit de la violence politico-religieuse. Faut-il y voir une certaine légitimation religieuse de la violence, comme le font plusieurs qui s'en réclament pour régler les affaires du monde, légitimation dont la théorie du sacrifice serait la matrice religieuse (Lempert 2000) ? Dans la mesure où la souffrance rédemptrice s'avère le fruit de la violence, on pourrait en effet croire que c'est aussi bien la violence qui est salutaire, comme le montrent à répétition les films de héros américains, chinois ou coréens, de même que le discours sacrificiel des bourreaux. Ce n'est certes pas là l'intention de l'Évangile qui valorise plutôt l'action divine à travers la geste de Jésus, la résurrection 
marquant la réponse de Dieu à la violence meurtrière, voire sa dissidence par rapport à cette violence. Mais on sait que la théologie de la rédemption a emprunté une autre route, préférant voir la main du Seigneur de l'histoire dans la souffrance (et la violence) de la croix elle-même, occultant en quelque sorte la violence derrière la valeur de la souffrance qui en résulte ou, au mieux, la spiritualisant comme péché.

\section{La mort et les souffrances de Jésus comme moyens de salut}

Avant de présenter les réflexions de ce numéro de Théologiques, rappelons les éléments majeurs des affirmations classiques qu'elles mettent en cause.

\subsection{Dans le Nouveau Testament}

On trouve dans le Nouveau Testament trois interprétations majeures de la mort de Jésus (Léon-Dufour 1979). La première, qui paraît la plus originelle, accorde peu d'importance à la mort de Jésus elle-même, mais s'intéresse plutôt à l'annonce du Seigneur et de l'action de Dieu qui ressuscite Jésus: "Celui que vous vous avez crucifié et que Dieu a ressuscité des morts» (Ac 4,10). Ce qui importe ici, ce n'est pas la mort de Jésus, qui est l'œuvre des hommes, mais le fait que Dieu l'a ressuscité et fait Seigneur (Ac 2,14-36).

La deuxième interprétation relève d'une communauté préoccupée de trouver un sens à la torture et à la mort du Seigneur. Dans la foulée de la conception de Dieu comme Seigneur de l'histoire, elle inscrit la mort et les souffrances de Jésus dans le dessein de Dieu — «Que ta volonté soit faite et non la mienne » $(\mathrm{Lc} 22,42)$ — et y réfléchit en fonction du thème de l'accomplissement des Écritures: Jésus a souffert et a été crucifié selon le plan de Dieu, tel qu'annoncé dans les Écritures. Mais le Nouveau Testament ne développe pas de réflexion systématique sur cette affirmation, contrairement à la théologie postérieure.

La troisième interprétation dit le sens et la portée de cette mort pour l'être humain. C'est à cause de nous, c'est pour nous et pour notre salut que le Christ est mort (Lc 22,19-20; Rm 4,25; Rm 5,8), comme don de Dieu lui-même «qui n'a pas épargné son propre Fils, mais l'a livré pour nous » ( $\mathrm{Rm} 8,32)$. Et encore, affirme l'évangile: «Le Fils de l'Homme est venu pour donner sa vie en rançon pour la multitude» (Mc 10,45; Mt 20,28), un énoncé qui en a amené plus d'un à considérer les évangiles comme «des récits de la Passion avec de longues introductions» (Kähler 1964, 80). 
C'est donc très tôt que la tradition considère la mort de Jésus comme un acte divin en notre faveur et non comme un acte fortuit ou comme le fruit de l'histoire humaine. Pour le croyant, Dieu agit dans l'histoire et de façon éminente au Golgotha et sur le chemin qui y mène.

\subsection{Les développements de la tradition}

Paul n'a pas systématisé les nombreuses images qu'il utilisait pour dire le sens de la mort de Jésus (Genest 1995). La tradition orientale a surtout développé l'affirmation de l'incarnation salvatrice, Dieu prenant notre humanité pour nous rendre participants de sa vie et le Christ récapitulant en lui l'histoire de l'humanité et de la création pour l'unir au Père dans l'Esprit. La tradition occidentale a plutôt mis l'accent sur le fonctionnement du salut, portée par une fascination pour la loi et la mécanique plus que pour la mystique. Trois métaphores majeures ont marqué cette réflexion sur le sens de la mort, puis de la souffrance du Christ: rançon, satisfaction, expiation.

\subsubsection{La mort de Jésus en rançon pour notre rachat}

La première de ces théories paraît la plus proche du Nouveau Testament: le Christ a donné sa vie en rançon pour la multitude. Le terme rançon traduit ici le grec lutron, un terme juridique concernant le rachat des prisonniers de guerre ou des esclaves. Parler de rançon, c'est dire que nous étions en captivité, en ce cas esclaves du péché, et que la mort du Christ nous en a délivrés. La rançon apparaît chez les Pères comme le prix payé pour notre rachat (comme on rachète encore des captifs de guerre dans certains pays d'Afrique ou des victimes d'enlèvement dans diverses régions du monde), le prix payé pour que l'homme retrouve son héritage divin. Mais cette rançon, à qui fut-elle payée ? se sont demandé les Pères, empêtrés dans le développement de la métaphore néotestamentaire. À Satan, répondirent les uns, à Dieu lui-même, répondirent les autres, non sans problèmes de part et d'autre. Quoiqu'il en soit, le rachat de l'humanité par la mort du Christ constitue l'objet même de l'Incarnation:

En définitive, écrit encore un théologien du début du $\mathrm{Xx}^{\mathrm{e}}$ siècle, si [le Christ] est venu sur terre, ce n'est pas pour guérir les malades, pour opérer des miracles, ni même pour prêcher le Royaume des Cieux. Tout cela n'est que l'extérieur, le côté visible de sa mission. Le vrai noyau central de son œuvre rédemptrice, c'est le rachat de notre vie par sa mort. (Deneken 1988, 89) 
À cette étape de la réflexion, on parle donc de don et non de châtiment ou d'expiation comme le feront les développements ultérieurs de la tradition.

\subsubsection{La mort de Jésus comme satisfaction et expiation}

Après la métaphore de la rançon vint celle de la satisfaction, systématisée par Anselme de Cantorbéry (1033-1109), qui allie toujours le juridique et l'économique. Une remarque s'impose cependant pour le lecteur non spécialisé. Le terme satisfaction ne désigne pas ici une satisfaction d'ordre psychologique, comme on le croit souvent, mais relève du droit et porte une notion d'équivalence. Satis facit, c'est faire assez. En ce cas, faire assez pour restaurer l'ordre du monde, la destinée originelle de l'homme et l'honneur de Dieu transgressés par le péché que Dieu, ne pouvant déroger aux lois de la justice, ne pouvait pardonner sans une réparation qui soit à la hauteur de l'offense. Comme on croit que l'agir humain ne peut y suffire, Anselme (Cur Deus homo) développe l'idée de la nécessité de l'incarnation et de la mort du Fils pour offrir à Dieu, au Père, la vie la plus précieuse qui soit, la seule capable de faire assez (satis facere) pour rétablir l'équilibre. On considère alors que l'éminence et la profondeur de la souffrance du Fils contribuent à la grandeur de la réparation et le salut apparaît comme la conséquence de ses souffrances et de sa mort librement consenties. Opère ici le principe de rétribution bien ancré dans l'Ancien Testament et que nous appuyons encore quand nous disons qu'il faut payer pour le mal qu'on a fait. En ce cas, toutefois, le Fils se substitue à l'humanité et paye à sa place. Prenant sur lui la faute et le châtiment de l'humanité, il les éloigne de nous. Une telle conception se situe non seulement dans l'ordre de la métaphysique, qui fait problème à la rationalité moderne, mais dans celui de la nécessité tout autant que dans celui de la grâce. Il s'agit de remettre une dette plutôt que de payer librement une rançon pour le rachat des captifs.

Avec le succès des idées d'Anselme se développent les représentations artistiques appuyant sur les souffrances du Christ, qui succèdent à l'iconographie représentant un Crucifié serein ou vainqueur. Le concile de Trente (1535-1545) durcira cette position en insistant encore davantage sur l'expiation et les souffrances qui y sont nécessaires et en développant la notion de sacrifice d'expiation dont la messe apparaît comme le mémorial sacramentel. 
Cette notion de sacrifice d'expiation s'inspire en partie de Paul ( $\mathrm{Rm}$ 3,21-26) et surtout de la Lettre aux Hébreux. Mais les exégètes nous mettent en garde contre une mauvaise compréhension de ces textes. Ainsi, Olivette Genest nous avertit que lire les textes du Nouveau Testament à valence sacrificielle

suppose une bonne connaissance de la conception propre au sacrifice chez les Hébreux [...]. S'appuyer, à l'inverse, sur le dictionnaire et la pratique de nos langues, où sacrifier sa vie signifie don de soi, et expiation signifie châtiment imposé ou offert pour réparer un manquement quelconque, entraîne la lecture dans l'ambiguité, voire le contresens. À titre d'exemple, dans la Bible hébraïque, l'expiation exprime l'enlèvement des péchés et non leur effacement par une réparation adéquate. Ce sont Dieu, et le prêtre qui performe le sacrifice, qui sont les sujets grammaticaux du verbe expier. En ce sens précis, c'est Dieu qui expie les péchés et non le coupable ou Jésus comme bouc émissaire. (Genest 1999)

Dans un article fort intéressant sur l'expiation, Shmuel Trigano montre que la kappara, que l'on traduit couramment par rachat ou rédemption, «ne concerne pas tant le rachat - et encore moins l'expiation [...] - des péchés que le rapprochement d'avec un Dieu caché, invisible et immatériel, dont la vision est mortelle» $(2002,119)$. L'offrande ou le sacrifice sert en fait de paravent pour le rapprochant, en le protégeant tant de la divinité que de son jugement éventuel. En fait, précise-t-il, «Le korbane/sacrifice n'occasionne pas une substitution mais une mise en mouvement» (123).

Nous n'avons pas ici le loisir de relire la Lettre aux Hébreux en fonction de ces riches remarques de Trigano. Mais on ne saurait arguer de sa sémantique sacrificielle pour considérer la souffrance de Jésus comme un moyen de satisfaction ou d'expiation au sens occidental du terme. Il n'est nullement question de souffrance dans le sacrifice rituel. C'est l'offrande rituelle confiante dans la miséricorde de Dieu qui est le moyen du sacrifice ou du rapprochement, fût-ce à travers l'aspersion du sang, et non la valeur en soi de cette offrande ou la souffrance, voire le coefficient de souffrance de l'offrande. Et comment dire que le bras du Seigneur aurait livré son Fils au sacrifice sur le Golgotha alors qu'il avait délivré Isaac du sacrifice sur le mont Moria - un sacrifice que la théologie chrétienne, témoignant de son parti pris, a vu comme préfiguration inachevée du sacrifice du Christ! Vraiment, le sacrifice et sa violence allaient bien à la théologie occidentale! 


\section{3 «La Passion du Christ» selon Mel Gibson, un témoin de la tradition}

Un an après sa sortie, le film La Passion du Christ (2004) était en septième position sur la liste des plus grands succès de l'histoire du cinéma, et son réalisateur au second rang des plus grandes fortunes du monde de l'entertainment américain. Ce succès, auquel a contribué le soutien des Églises, nous semble très significatif, particulièrement pour un film dont on a partout souligné la violence. Or, on ne peut que remarquer la parenté de ses images avec les représentations graphiques des souffrances du Christ qui avaient marqué la fin du Moyen Âge et la spiritualité doloriste de l'époque moderne. Alors que la modernité avait remplacé la punition et le supplice publics par la prison où le redressement, la surveillance et le contrôle se substituant à la mise en scène publique de la souffrance de l'expiation (Foucault 1975), le dolorisme s'était au contraire attaché à la mise en scène classique du supplice et de la douleur qu'a retrouvée La Passion $d u$ Christ - comme l'ont aussi retrouvée Hollywood, certains gouvernements islamistes, les terroristes vidéastes et les nouvelles télévisées.

La souffrance, on le sait, occupe une place centrale dans le film de Gibson. Toute la place, ont dit plusieurs. Or, cette souffrance est présentée à Gethsémani comme volonté de Dieu, alors que le film montre bien qu'elle est le fruit de la violence des bourreaux. De plus, elle est pratiquement recherchée par le héros qui, dans la tradition américaine des films de sauveurs d'abord brutalisés à laquelle a souvent participé Mel Gibson comme comédien et réalisateur, semble en redemander alors qu'il provoque du regard les soldats qui viennent de ranger leurs fouets. Même imaginaire du héros hollywoodien, alors que la résurrection est soulignée par une musique victorieuse, quasi militaire, et des images qui évoquent la puissance d'un Schwarzenegger dans Commando ou Terminator.

Le plus troublant du film de Gibson relève peut-être de son rapport traditionnel à la volonté de Dieu. La majorité des protagonistes du film (et de la Passion) paraissent en effet au service de la volonté de Dieu que Jésus a acceptée à Gethsémani: les prêtres et les grands prêtres l'amènent à Pilate, les soldats de l'Empire le flagellent et le torturent, Pilate le condamne, les soldats, encore, le mènent à la croix — instrument de notre salut — et l'y crucifient. Sans eux, point de salut, et l'enfer ne se viderait pas à la mort de Jésus. Tous travaillent donc à la volonté de Dieu devant les yeux de foules plus ou moins passives et du diabolique androgyne voyeur qui paraît ignorer qu'il court en réalité à sa perte. 
Il n'y a que la femme de Pilate pour tenter de faire échec à la volonté de Dieu en suggérant à son époux de libérer Jésus. Certes, on n'a pas coutume de lire ainsi le film de Gibson ou l'évangile, et la femme de Pilate y apparaît sympathique à Jésus. Mais son rôle y est néanmoins ambigu et porte à réfléchir. On pourrait même dire qu'elle risque de nuire à la mécanique du salut en marche dans le récit. Ne voit-on pas que si elle réussit à convaincre Pilate de libérer Jésus, c'est nous qui ne le serons pas, c'est le projet de Dieu qui ne pourrait se réaliser? Cela paraît insensé, mais c'est pourtant bien là la conséquence d'une position qui considère la souffrance et la mort de Jésus comme rédemptrices, la conséquence d'une théologie qui tient pour volonté de Dieu la Passion, les souffrances et la mort de Jésus. Dans un tel système, où la défense de l'innocent risque de faire échec au plan divin, l'injustice et la torture deviennent non seulement moyens, mais conditions du salut. Dans une telle théologie, pas de violence, pas de salut! Le salut paraît carburer à la soumission de l'innocent, à la souffrance, au sang et à la violence.

\subsection{Une relecture contemporaine}

Heureusement, ces interprétations classiques n'ont pas que des aspects négatifs et malgré leurs excroissances perverses, on retiendra aussi qu'elles parlent d'abord du salut. D'un salut dans lequel c'est toujours l'humanité qui se trouve bénéficiaire du don de Dieu et de son Fils. Un salut dont Dieu luimême paye le prix, que Bonhoeffer désignait comme «le coût de la grâce». Or, nous savons que la justice et la libération (salut) ont toujours un prix, un coût que bien des hommes et des femmes payent encore malheureusement chaque jour et dont certains trouvent soutien dans la geste de Jésus.

Mais ce soutien peut prendre d'autres voies. La théologie contemporaine considère autrement la mort de Jésus que ne l'ont fait les époques précédentes: non comme le fait de Dieu qui livrerait son Fils à la torture, mais, dans une perspective d'abord historique, comme celui des hommes et des pouvoirs qu'il a mis en cause. Avec l'Incarnation, c'est à la vie que le Fils est livré, la vie dont la mort et les risques de violence font évidemment partie. C'est par sa vie, sa mort et sa résurrection, c'est par son Esprit présent parmi nous que Jésus sauve. Contrairement aux siècles passés, on dira aujourd'hui que «Christ vient dans la chair attester de la vérité de Dieu et de la vérité de l'homme et il en meurt. Il ne vient pas pour mourir» (Gisel 1980, 148). C'est l'amour de Dieu qui sauve et non la mort et la souffrance... que cet amour rencontre lorsqu'il s'incarne dans notre histoire. 
En cela, la souffrance et la croix ne sont en rien étrangères au salut, puisqu'elles ne sont en rien étrangères à notre existence et à notre histoire. Mais non par quelque nécessité métaphysique.

Jon Sobrino, dont les confrères de l'université de San Salvador ont été assassinés par des militaires salvadoriens en 1989, considère la croix comme lieu de crédibilité de Dieu pour ceux qui souffrent $(1978,226)$. Il est de ceux et celles qui croient que c'est en plongeant dans les horreurs de cette histoire, en subissant les conséquences d'une participation intime au processus de protestation et de combat contre le mal que Dieu attire à lui le monde et son histoire (272). Avec la résurrection, se trouve ouverte, pour ceux et celles qui sont crucifiés par l'histoire, la possibilité d'aller à ce Dieu qui ressuscite Jésus. S'identifiant avec ce qui semble perdu, Dieu affirme dans la résurrection, comme il le faisait déjà dans la pratique de Jésus, qu'il n'abandonne pas ce qui est perdu, qu'il n'abandonne pas ceux et celles qui sont torturés dans l'histoire. Et non que leur torture advient selon sa volonté. C'est ainsi que la nouvelle orthodoxie théologique contemporaine du Dieu souffrant, quoiqu'il en soit de son caractère apologétique, s'inscrit dans les paradigmes de l'impuissance et de l'herméneutique des victimes et non plus, ou non plus seulement, dans ceux de l'expiation et de la substitution.

\section{Souffrances rédemptrices de Jésus et sens de la souffrance humaine}

Si la souffrance de Jésus n'est pas en tant que telle l'effet de la volonté de Dieu, si elle n'a pas en tant que telle une valeur rédemptrice, qu'en est-il alors des souffrances humaines dont la tradition affirme qu'elles y participent (Jean-Paul II 1984) ? Le problème se pose d'autant plus que la réflexion sur la mort et la résurrection de Jésus s'est dès le début développée en fonction de notre participation à cette mort-résurrection. La question est importante aussi au regard de notre préoccupation pour le sens et la valeur de nos souffrances. Combien de fois entend-on les parents d'une victime d'assassinat, par exemple, réclamer que la mort et la souffrance de leur proche aient un sens, fût-ce dans la prévention d'autres morts semblables?

Trouver sens à la souffrance ou réinterpréter celle-ci constitue un facteur important, en même temps qu'un indice de résilience chez les victimes de violence. Mourir sans raison! Subir une mort ou une souffrance insensée! Voilà ce qui fait problème. Viktor E. Frankl (1985) a bien montré à partir de son expérience des camps de la mort nazis comment le fait de donner sens à la souffrance permet de la porter. Le sens apaise, écrit Ricœur 
$(1988,62)$, alors que Jüngel constate que donner sens à la souffrance contribue à la rendre supportable. Distinguant explication et compréhension de la souffrance, Jüngel écrivait que «the explanation does not make the pain more bearable» $(1988,4)$. D'où la valeur existentielle de la compréhension de la souffrance de Jésus comme rédemptrice et de la participation des souffrances humaines à cette souffrance. On dira alors que le concept de souffrance rédemptrice a le mérite de sauver la souffrance elle-même... et ceux qui souffrent. La souffrance salvatrice (et donc sensée) est ellemême une souffrance sauvée. Le concept de souffrance rédemptrice permet d'inverser le signe d'emblée négatif de la souffrance. Il le fait d'abord pour le Christ, mais il le fait aussi pour les croyantes et les croyants: «Crucifiés avec lui, nous ressusciterons avec lui » (voir Rm 6,8; 2Co 13,4; $2 \operatorname{Tm} 2,11)$.

Ce versant positif de la tradition est lourdement assombri par son versant négatif qui paraît légitimer la souffrance. Dans la tradition chrétienne, il a souvent semblé à plusieurs croyants comme à des non-croyants que le concept de souffrance rédemptrice ne sauvait pas seulement la souffrance ou la valeur, voire la dignité du souffrant, mais qu'il servait trop souvent à justifier la souffrance en maintenant la victime dans sa situation, encourageant sa soumission aussi bien que l'inaction de spectateurs silencieux malgré la tradition caritative très forte en christianisme. Plus critique et plus sensible aux dynamiques de l'histoire, la théologie contemporaine a pris conscience de l'effet paralysant que ce discours a parfois eu sur les victimes de l'histoire (esclaves, Noirs, femmes, pauvres, victimes de violence familiale, etc.). Le reproche est bien connu qui se résume souvent en celui de favoriser la résignation à une situation de violence, d'oppression ou d'exploitation. Que la situation soit individuelle ou collective. Mais tout dépend de la relation que l'on établit entre Dieu et cette souffrance. Une souffrance fruit de la volonté ou du plan de Dieu a un autre statut qu'une souffrance que Dieu assume et, Maître du grand œuvre, transforme en bien. Un Dieu qui ressuscite est autre qu'un Dieu qui livre à la croix. Depuis Kant, en effet, la pensée - et parfois l'agir - se réclame des maximes selon lesquelles on doit traiter autrui comme une fin et non comme un moyen... et que la fin ne justifie pas les moyens.

Plusieurs théologiens et théologiennes ont donc critiqué les effets pervers d'une théologie de la rédemption basée sur la valeur de la souffrance et sur la soumission de Jésus à la volonté de son Père. Mais le problème reste entier pour ceux et celles qui souffrent et ne peuvent qu'assumer cette 
souffrance, dépourvus de moyens de la réduire ou d'en combattre les causes, ou encore pour ceux et celles dont "leur» souffrance est tout ce qu'il leur reste, comme on le dit parfois. La croix du Christ est une grâce réelle pour ceux et celles qui continuent d'unir leurs souffrances à celles du Christ, selon le modèle de la participation des souffrances humaines aux souffrances salvifiques du Christ, leur donnant ainsi sens et valeur. Ce concept de la participation aux souffrances du Christ permet en effet de donner sens aux souffrances insensées. C'est là sa noblesse, une noblesse qu'il faut retrouver, avec le sens, la dignité et l'espérance qu'elle porte pour ceux et celles qui souffrent, même si le caractère rédempteur de ces souffrances pose problème. C'est bien plus la résurrection que la valeur rédemptrice de la souffrance qui s'avère porteuse d'espérance pour ceux et celles qui souffrent. C'est sur la grâce de la résurrection que doivent porter nos réflexions. Se révèle là un Dieu différent du Souverain détenant et brandissant le pouvoir, intervenant dans l'histoire de l'extérieur de celle-ci. Participant jusqu'au bout à cette histoire, Dieu connaît la souffrance qui marque notre fragilité, nos combats, notre devenir. La croix nous révèle que l'amour de Dieu ne nous donne pas seulement le monde ou un plan pour y vivre, mais qu'il se donne lui-même (McDonagh 1979).

\section{Les contributions de ce numéro}

Ce numéro de Théologiques voudrait contribuer à la vaste tâche de revoir le discours sur la valeur rédemptrice ou salutaire de la souffrance, d'abord dans le christianisme mais aussi dans les autres monothéismes. Il s'ouvre avec un article de Jean-Marc Gauthier qui pose directement la question à sa source en soulignant le scandale pour la sensibilité contemporaine nordoccidentale de la perspective classique du salut par la souffrance et la mort du Christ, et donc par la violence qui lui est liée. Dans une réflexion libre, mais nourrie par des années de lecture et de réflexion sur ces questions, Gauthier part de la place centrale de la Passion et de la mort de Jésus dans la révélation et la manifestation du salut. Il se demande s'il est "possible de penser la foi chrétienne, du dedans de cela, tout en remettant en question tout lien de cause à effet entre violence et rédemption, entre souffrance et rédemption, entre mort et rédemption... ou salut». Ce qui, on l'avouera, ne manque pas d'audace de la part de quelqu'un qui reconnaît qu'il s'agit ici de l'essentiel de la foi chrétienne et qui continue d'affirmer la place centrale de la croix dans l'histoire du salut. Si la croix représente ce dont il nous faut être sauvés, comment alors saisir que le salut y advient tout de même? 
Suit une entrevue avec Shmuel Trigano, directeur de la revue Pardès, dont nous avions apprécié le dossier sur Le sacrifice $d u$ fils dans les trois monothéismes (Trigano et al. 1996). Nous voulions interroger M. Trigano sur la valeur rédemptrice de la souffrance en judaïsme, particulièrement quant à la Shoab et aux interprétations qui lui trouvent sens dans le plan divin en tant que châtiment, épreuve ou moyen pour diverses fins. $\mathrm{Ou}$ encore sur le sens de la souffrance des martyrs d'Israël, depuis les Macchabée en passant par les martyrs de Massada jusqu'aux martyrs de la Shoah. Or, dans ces cas, prévient Trigano, ce n'est pas la souffrance qui a valeur, mais le témoignage de la foi. Trigano reconnaît néanmoins dans le judaïsme l'existence d'une mystique de la souffrance, voire d'un discours de la souffrance pour la sanctification du nom (Kidouch haChem), mais elle n'y est pas majoritaire et n'y a pas le poids de la souffrance rédemptrice en christianisme. Pour Trigano, la souffrance du Serviteur, comme celle du peuple qu'il représente, est un scandale, un scandale que nivelle le fait de lui donner sens. Reste la question de la violence et de sa présence dans la Bible, voire même du côté de Dieu. Trigano soutient que cette présence permet de prendre en compte la place de la violence dans l'existence humaine, mais sur le plan historique, il invite à chercher la responsabilité humaine et non la responsabilité divine là où il y a un acte humain. Ce qu'il fait devant la violence au Moyen-Orient, comme devant la Shoah dont l'horrifient les interprétations religieuses qui en attribuent à Dieu quelque dessein ou responsabilité.

Dans une perspective de sciences politiques, Ali G. Dizboni réfléchit à la question de la souffrance et de la violence dans l'islam. Il explore le statut du martyr et la signification du sacrifice physique en islam sur le fond du concept de diihad dans le Coran et les hadiths. En fait, Dizboni considère le martyre et le djihad comme les deux faces d'une même médaille dont il montre bien les développements historiques en fonction de leur contexte. Marquée par l'audace d'une réflexion qui fait dialoguer les textes et l'histoire, sa contribution examine plus spécifiquement certains aspects rituels, politiques et idéologiques du martyre dans l'islam, particulièrement dans le chiisme. Ce faisant, il nous offre des outils intéressants non seulement pour la réflexion, mais aussi pour la connaissance du contexte et des enjeux contemporains de cette tradition.

L'article suivant est le fruit d'une collaboration de recherche entre JeanGuy Nadeau, Carole Golding et Claude Rochon. Il s'intéresse aux effets de la figure de la Passion de Jésus sur les victimes d'abus sexuels. La Passion 
constitue en effet pour la foi chrétienne une ressource majeure pour faire face à la souffrance et à la violence. Or, la figure de la Passion paraît ambiguë dans des contextes de victimisation, dont celui des abus sexuels en milieu familial, un phénomène qui touche une large partie de la population. Si l'éducation religieuse de l'enfant peut l'aider à faire sens de ce qui lui arrive et à le surmonter, elle peut aussi aggraver son sort en le soumettant davantage à son agresseur, voire en l'incitant à voir la volonté de Dieu dans son expérience. Si les injonctions à l'imitation de Jésus Christ peuvent s'avérer délétères pour la foi de l'enfant, l'expérience d'identification au Crucifié peut aussi permettre aux victimes de se sentir comprises par Dieu et de s'identifier au Ressuscité. Enfin, l'article signale de nouvelles approches théologiques qui valorisent davantage la résistance de Jésus que sa soumission.

À partir du cadre méthodologique de la biographie comme lieu théologique, Armel Brice Adanhounme propose de saisir la mémoire de l'esclavage des Noirs d'Afrique comme un possible capital de rédemption. Il explore particulièrement la vie de sainte Joséphine Bakhita, ancienne esclave africaine chez qui il voit une dynamique de libération des chaînes de l'esclavage. À partir de l'hagiographie de la sainte, il suggère que trois points - le récit, le souvenir et la récupération de la mémoire de l'esclavage structurent le paradigme d'une théologie de l'esclavage chez Bakhita. Il voit dans ce récit la subversion historique et herméneutique du récit hégélien sur l'esclavage des Noirs et une rébellion prophétique par la possibilité de dire non.

Dans son article sur le film de Mel Gibson, La Passion du Christ, Michel-M. Campbell s'intéresse tout autant à la médiatisation et à la réception du film qu'à sa facture et à son contenu. Il rapporte une partie des discussions, des appréciations (ecclésiales) et des mises en garde (théologiques) vis-à-vis de ce film. Ce qui en ressort, c'est la relation particulière du public et des Églises à un film vu et discuté par des dizaines de millions de personnes à travers le monde, surtout occidental, une relation qui s'enracine en partie dans le rapport à la souffrance du Christ mise en scène par le film de Gibson.

Notre numéro se clôt avec un texte de René Girard. À l'occasion de l'obtention d'un doctorat honorifique de l'Université de Montréal au printemps 2004, il a prononcé une conférence dans laquelle il rappelait les grandes lignes de la théorie mimétique et de sa théorie du bouc émissaire. Il y rappelait que la Passion de Jésus et les évangiles qui en rendent comptent démystifient et démythisent la violence sacrificielle et rédemptrice. La 
revue Théologiques l'a invité à publier cette conférence. Il a préféré publier le texte que l'on retrouve ici, texte où il résume ses thèses sur la question et surtout son rejet de l'assimilation de la Passion au religieux archaïque, assimilation qu'il dénonce comme "la colonne vertébrale du nihilisme moderne».

Nous avons voulu ce numéro ouvert sur diverses perspectives et traditions religieuses. Les contraintes de l'édition ont fait que manquent de larges pans de la réflexion, particulièrement un exposé de la valeur contemporaine du concept de souffrance rédemptrice. Mais comme ce concept est bien connu et que nous y avons fait écho dans les pages précédentes, il nous a semblé que nous pouvions publier les réflexions qui suivent et espérer que le lecteur y trouvera matière à réflexion et à discussion.

\section{Références}

Deneken, M. (1988), Le salut par la croix dans la théologie catholique contemporaine, Paris, Cerf.

Foucault, M. (1975), Surveiller et punir. Naissance de la prison, Paris, Gallimard (Bibliothèque des Histoires).

Frankl, V.E. $\left(1985^{3}\right)$ [1962², 1959, allemand 1946], Man's Search for Meaning, New York, First Washington Press.

Genest, O. (1995), Le discours du Nouveau Testament sur la mort de Jésus. Épîtres et Apocalypse, Sainte-Foy, Presses de l'Université Laval.

- (1999), «L'actualisation de la mort de Jésus et du sacrificiel », Science et Esprit, 52, p. 49-64.

Gisel, P. (1980), La création, Genève, Labor et Fides.

Jean-Paul II (1984), Le sens chrétien de la souffrance humaine / lettre apostolique Salvifici Doloris, Montréal / Paris, Fides / Cerf.

JÜNGEL, E. (1988), "The Christian Understanding of Pain », Journal of Theology for Southern Africa, 65, p. 3-13.

KäHLER, M. (1964) [allemand 1896], The So-Called Historical Jesus and the Historical Biblical Christ / trad. par C.E. Braaten, Philadelphia, Fortress Press.

Lempert, B. (2000), Critique de la pensée sacrificielle, Paris, Seuil.

LÉON-Dufour, X. (1979), «La mort rédemptrice du Christ selon le Nouveau Testament ", dans X. LÉon -Dufour et al., Mort pour nos péchés, Bruxelles, Facultés universitaires Saint-Louis, p. 14-44. 
McDonagh, E. (1979), "La dignité de Dieu et la dignité de l'homme ", Concilium, 150, p. 137-147.

Riceur, P. (1988), «Le scandale du mal», Esprit, 7/8, p. 57-63. (1991) «Événement et sens", dans J.L. Petit, L'événement en perspective, Paris, Éditions de l'École des Hautes Études en Sciences Sociales, p. 41-56.

Sobrino, J. (1978), Christology at the Crossroads. A Latin American Approach, Maryknoll, Orbis Books.

(1986), «La foi dans le Fils de Dieu à partir d'un peuple crucifié », dans Jésus en Amérique latine. Sa signification pour la foi et la christologie, Paris, Cerf (Cogitatio Fidei 140).

Trigano, S. (2002), «Le bouc émissaire. En finir avec l'expiation », Pardès, 32-33: La Bible et l'Autre, p. 117-127.

Trigano, S. et al. (1996), Pardès, 22: Le sacrifice du fils dans les trois monothéismes. 